\title{
Least Significant Bit Steganography using Hitzl-Zele Chaotic Map
}

\author{
Krasimir Kordov and Borislav Stoyanov
}

\begin{abstract}
We propose a novel least significant bit steganography algorithm based on a Hitzl-Zele chaotic function. On the first step a pseudorandom generator is constructed for chaotic pixel selection for hiding the secret message. Exact study has been provided on the novel scheme using visual inspection, peak signal-to-noise ratio, and histogram analysis. The experimental data show excellent performance of the novel stego technique.
\end{abstract}

Keywords-Steganography, Steganalysis, Chaotic functions, Pseudorandom number generator.

\section{INTRODUCTION}

$\mathbf{S}$ TEGANOGRAPHY is art and science of hiding secret information (message) in plain sight without being noticed within an innocent cover data (container) so that it can be securely broadcasted over a communications channel. First used containers usually were hand written text, images ets. but with technology progress one of the most used containers these days are raster graphic files.

In recent years, with rapid development of information and communication technologies, web-based services (tools) are becoming increasingly favourite. Steganography, as a part of science of securely data transmission [6], [20], is an art of inconspicuous message sending. There is a need for new stego algorithms, that provide the necessary security and privacy in data communication [29]. Using chaotic maps in steganography algorithms become more popular, because of the resistance of increasing stego attacks.

In two fundamental papers [15], [16] F. Pichler and J. Scharinger proposed the chaotic Baker map to securing digital communications. J. Fridrich in [9] extended their work by adapting nonlinear two-dimensional maps on a torus and on a square, with aims to achieve better encryption.

Least significant bit (LSB) method based on Henon map is presented in [14]. In [17], novel least significant bit method based on 1D logistic map is proposed. In [12], a novel steganographic algorithm using Baker and Logistic maps is designed. Two-level steganographic methods based on chaotic maps are presented in [18] and in [13]. Novel LSB hiding algorithm, which uses chaotic rotation equations, is proposed in [26].

In Section II we propose a novel pseudorandom bit generator based on Hitzl-Zele chaotic function. In Section III we present a novel LSB image steganography algorithm,

This work is supported by the Scientic research fund of Konstantin Preslavski University of Shumen under the grant No. RD-08-124/06.02.2017.

Krasimir Kordov and Borislav Stoyanov are with the Department of Computer Informatics, Konstantin Preslavsky University of Shumen, 9712 Shumen Bulgaria, e-mails: krasimir.kordov@shu.bg, borislav.stoyanov@shu.bg. and extended performance analysis is given. Finally, the last section concludes the paper.

\section{PSEUDORANDOM BIT GENERATOR BASED ON HITZL-ZELE CHAOTIC MAP}

Pseudorandom generators are basic primitives used in cryptography algorithms but in our case we apply the random properties of chaotic pseudorandom bit generator to steganography algorithm. Pseudorandom generators are software realized methods for extracting sequence of random values.

\section{A. Proposed Pseudorandom Bit Generation Algorithm}

The Hitzl-Zele function [10], [19], is a three-dimensional discrete-time dynamical system given by:

$$
\begin{aligned}
& x(k+1)=1+y(k)-z(k) \cdot x^{2}(k) \\
& y(k+1)=a \cdot x(k) \\
& z(k+1)=b \cdot x^{2}(k)+z(k)-0.5,
\end{aligned}
$$

with bifurcation parameters $a=0.25$ and $b=0.87$ for chaotic behaviour. Figure 1(a), Figure 1(b) and Figure 1(c) represents graphic plotting points of $x, y$ and $z$ dimensions.

We propose a new pseudorandom bit generation algorithm with the following steps:

Step 1: The initial values $x(0), y(0)$, and $z(0)$ from Eq. (1), and a bit stream limit $L$ are determined.

Step 2: The Hitzl-Zele chaotic map is iterated initially for $L$ times.

Step 3: The iteration of the Eq. (1) continues, and as a result, three real fractions $x(i), y(i)$, and $z(i)$, are calculated.

Step 4: The first two numbers $x(i)$ and $y(i)$ are postprocessed as follows:

$$
\begin{aligned}
& h_{0}=a b s(\bmod (\text { integer }(x(i) \times 10000000), 2) \\
& h_{1}=\operatorname{abs}(\bmod (\operatorname{integer}(y(i) \times 10000000), 2),
\end{aligned}
$$

where $a b s(x)$ returns the absolute value of $x$, integer $(x)$ returns the the integer part of $x$, truncating the value at the decimal point, $\bmod (x, y)$ returns the reminder after division.

Step 5: Perform XOR operation between $h_{0}$ and $h_{1}$ to get a single output bit.

Step 6: Return to Step 3 until the bit stream limit is reached.

The presented pseudorandom bit generator is implemented in $\mathrm{C}++$, using the following initial values: $x(0)=0.9134$, $y(0)=0.6324, z(0)=0.0975$, and $L=550$.

Similar chaos based PRG can be found in [3], [5], [7], [8], [22], [23], [24], [25], [27]. 


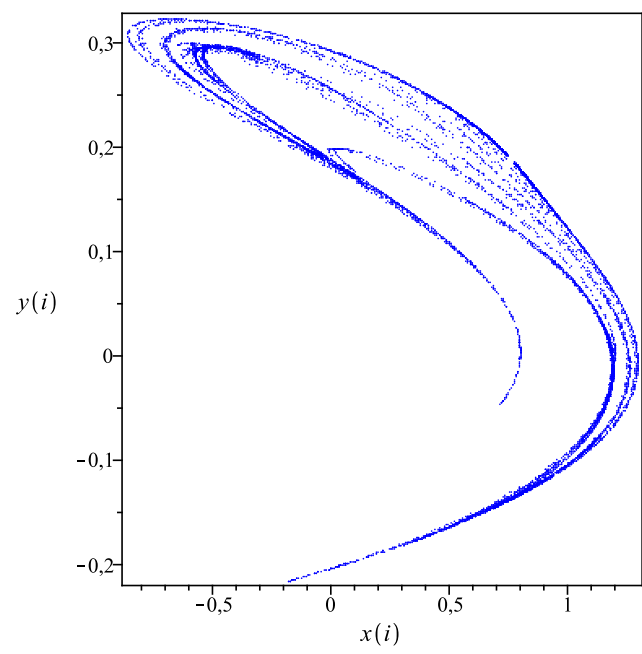

(a) 2D plot of the Hitzl-Zele chaotic function using $x$ and $y$ values.

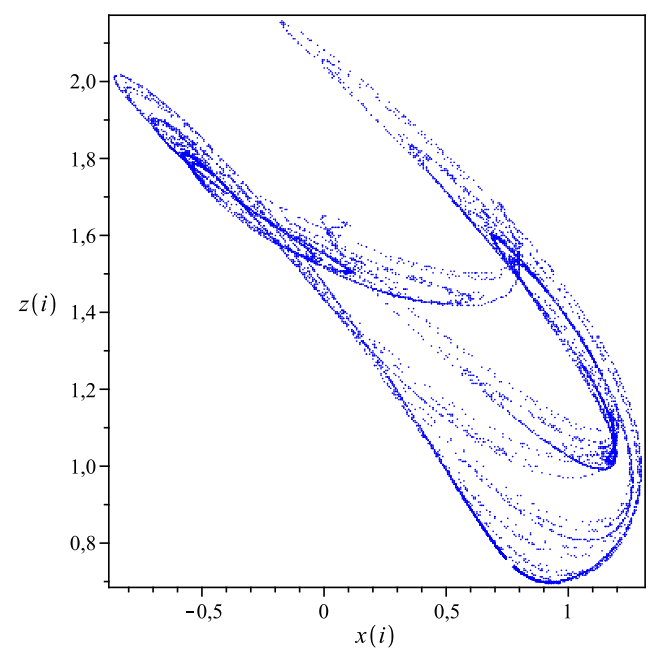

(b) 2D plot of the Hitzl-Zele chaotic function using $x$ and $z$ values.

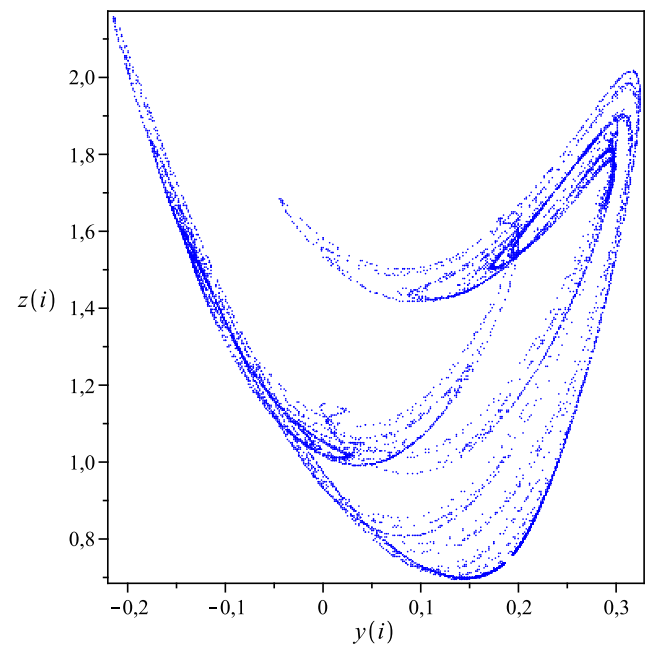

(c) 2D plot of the Hitzl-Zele chaotic function using $y$ and $z$ values.

Fig. 1. 2D plot of the Hitzl-Zele chaotic function

\section{B. Key space}

The key space is configured by the four numbers $x(i), y(i)$, and $z(i)$, and $L$. With number of about 16 decimal digits precision [30] the key space is more than $2^{172}$. This is large enough against exhaustive attack methods [1].

\section{Key sensitivity}

Testing key sensitivity requires comparison of result output binary sequences using very similar secret keys. In order to do the experiments we changed a single digit of variables from key space of the proposed pseudorandom generator. The secret key 1 uses the initial values described in Section II, the secret key 2 is formed by changing $x(0)$ to 0.9135 , for secret key 3 $x(0)$ is changed to 0.9324 , for secret key $4 y(0)$ is changed to 0.6325 , for secret key $5 y(0)$ is changed to 0.6334 , and for the last two secret keys 6 and 7, $z(0)$ is changed to 0.0976 and 0.0985 respectively. The next figures visually show the result sequences calculated using different but similar secret keys.

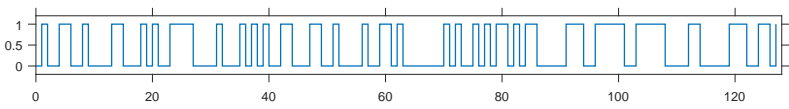

(a) Plot of binary sequence using secret key 1 .

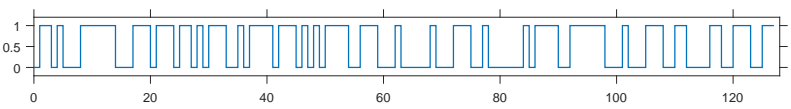

(b) Plot of binary sequence using secret key 2 .

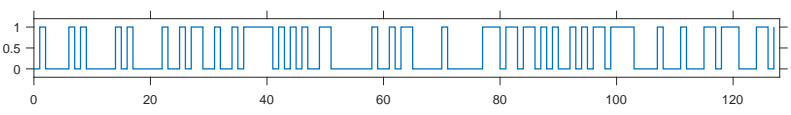

(c) Plot of binary sequence using secret key 3 .

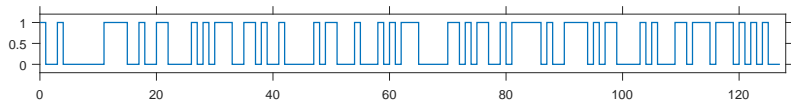

(d) Plot of binary sequence using secret key 4 .

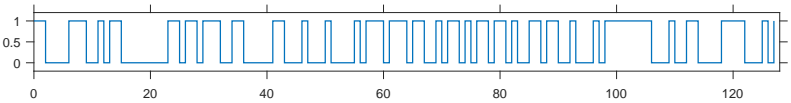

(e) Plot of binary sequence using secret key 5 .

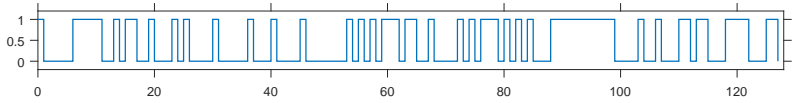

(f) Plot of binary sequence using secret key 6

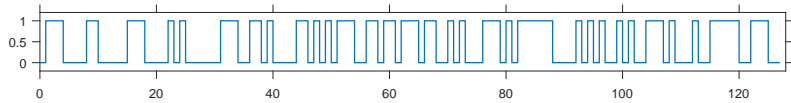

(g) Plot of binary sequence using secret key 7.

Fig. 2. Plot of binary sequences with similar keys.

All the figures are very different, demonstrating that even if a single digit is changed from the initial values of $x(0), y(0)$ or $z(0)$, the end result is entirely changed. The results indicates high level of security provided by the proposed pseudorandom generator as far as the secret key is concerned. 


\section{Statistical tests}

Three software test programs are used in order to measure the behaviour of the output binary streams.

The NIST test suite [21] includes 15 statistical tests: Frequency (monobit), Block-frequency, Cumulative sums (forward and reverse), Runs, Longest run of ones, Rank, Fast Fourier Transform (spectral), Non-overlapping templates, Overlapping templates, Maurers "Universal Statistical", Approximate entropy, Random excursion, Random-excursion variant, Serial, and Linear complexity. The proposed pseudorandom bit generator passed successfully NIST test suite, Table I.

TABLE I

NIST TEST SUITE RESULTS.

\begin{tabular}{|l|c|c|}
\hline \multirow{2}{*}{ NIST } & \multicolumn{2}{|c|}{ Proposed Algorithm } \\
\cline { 2 - 3 } statistical test & P-value & Pass rate \\
\hline Frequency (monobit) & 0.709558 & $994 / 1000$ \\
Block-frequency & 0.473064 & $991 / 1000$ \\
Cumulative sums (Forward) & 0.753844 & $993 / 1000$ \\
Cumulative sums (Reverse) & 0.624627 & $994 / 1000$ \\
Runs & 0.844641 & $989 / 1000$ \\
Longest run of Ones & 0.526105 & $994 / 1000$ \\
Rank & 0.433590 & $990 / 1000$ \\
FFT & 0.723804 & $990 / 1000$ \\
Non-overlapping templates & 0.477861 & $990 / 1000$ \\
Overlapping templates & 0.504219 & $986 / 1000$ \\
Universal & 0.094285 & $987 / 1000$ \\
Approximate entropy & 0.997698 & $988 / 1000$ \\
Random-excursions & 0.567112 & $634 / 641$ \\
Random-excursions Variant & 0.509168 & $634 / 641$ \\
Serial 1 & 0.000976 & $988 / 1000$ \\
Serial 2 & 0.128874 & $987 / 1000$ \\
Linear complexity & 0.370262 & $988 / 1000$ \\
\hline The minimum pass rate for each statistical test with \\
the exception of the random excursion (variant) test \\
is approximately = 980 for a sample size = 1000 \\
binary sequences. The minimum pass rate for the \\
random excursion (variant) test is approximately 627 \\
for a sample size 641 binary sequences. \\
\hline \multicolumn{2}{|c|}{} \\
\hline
\end{tabular}

The DIEHARD package [11] is set of 19 statistical tests: Birthday spacings, Overlapping 5-permutations, Binary rank (31 x 31), Binary rank (32 x 32), Binary rank (6 x 8), Bitstream, Overlapping-Pairs-Sparse-Occupancy, OverlappingQuadruples-Sparse-Occupancy, DNA, Stream count-the-ones, Byte-count-the-ones, Parking lot, Minimum distance, 3D spheres, Squeeze, Overlapping sums, Runs (up and down), and Craps. The tests return $P$-values, which should be uniform in $[0,1)$, if the input file contains pseudorandom numbers. The $P$-values are obtained by $p=F(y)$, where $F$ is the assumed distribution of the sample random variable $y$, often the normal distribution. The proposed pseudorandom bit generator passed successfully DIEHARD tests, Table II.

The ENT package [28] includes 6 tests to pseudorandom sequences: Entropy, Optimum compression, $\chi^{2}$ distribution, Arithmetic Mean value, Monte Carlo Value for $\pi$, and Serial Correlation Coefficient. The sequences of bytes are stored in files. The suite outputs the results of those tests. We tested output sequences of 125000000 bytes of the novel pseudorandom bit generation algorithm. The novel pseudorandom bit generation algorithm passed successfully ENT test, Table III.

From key space evaluation and the positive test results, we can conclude that the novel pseudorandom bit generation
TABLE II

DIEHARD STATISTICAL TEST RESULTS.

\begin{tabular}{|c|c|}
\hline $\begin{array}{l}\text { DIEHARD } \\
\text { statistical test }\end{array}$ & $\begin{array}{c}\text { Proposed Algorithm } \\
\text { P-value }\end{array}$ \\
\hline Birthday spacings & 0.393909 \\
\hline Overlapping 5-permutation & 0.518083 \\
\hline Binary rank $(31 \times 31)$ & 0.346194 \\
\hline Binary rank $(32 \times 32)$ & 0.340976 \\
\hline Binary $\operatorname{rank}(6 \times 8)$ & 0.561724 \\
\hline Bitstream & 0.499964 \\
\hline OPSO & 0.669539 \\
\hline OQSO & 0.578114 \\
\hline DNA & 0.635990 \\
\hline Stream count-the-ones & 0.047321 \\
\hline Byte count-the-ones & 0.452763 \\
\hline Parking lot & 0.612213 \\
\hline Minimum distance & 0.486939 \\
\hline 3D spheres & 0.638137 \\
\hline Squeeze & 0.057748 \\
\hline Overlapping sums & 0.533742 \\
\hline Runs up & 0.571370 \\
\hline Runs down & 0.236529 \\
\hline Craps & 0.357239 \\
\hline
\end{tabular}

TABLE III

ENT STATISTICAL TEST RESULTS

\begin{tabular}{|c|c|}
\hline $\begin{array}{l}\text { ENT } \\
\text { statistical test }\end{array}$ & $\begin{array}{l}\text { Proposed Algorithm } \\
\text { results }\end{array}$ \\
\hline Entropy & 7.999998 bits per byte \\
\hline Optimum compression & $\begin{array}{l}\text { OC would reduce the size of } \\
\text { this } 125000000 \text { byte file } \\
\text { by } 0 \% .\end{array}$ \\
\hline$\chi^{2}$ distribution & $\begin{array}{l}\text { For } 125000000 \text { samples is } \\
415.50 \text {, and randomly would } \\
\text { exceed this value } 0.01 \% \\
\text { of the time. }\end{array}$ \\
\hline Arithmetic mean value & $127.5004(127.5=$ random $)$ \\
\hline Monte Carlo $\pi$ estim. & 3.141717554 (error $0.00 \%$ ) \\
\hline Serial correl. coeff. & $\begin{array}{l}0.000012 \\
\text { (totally uncorrelated }=0.0 \text { ) }\end{array}$ \\
\hline
\end{tabular}

algorithm has good statistical properties and provide high level of security and randomness.

\section{LEASt SignificANT Bit SteganogRAphy USING HITZL-ZELE CHAOTIC MAP}

\section{A. Proposed Least Significant Bit Steganography Algorithm}

Here we design a novel LSB image steganography scheme by using the pseudorandom bit generation algorithm based on the Hitzl-Zele chaotic function, Section II.

We consider plain and stego images of $n \times m$ size. The bytes of the BMP pixel's grid are randomly passed based on HitzlZele pseudorandom bit generation. The header information bits are directly transferred into the stego image.

The novel steganography algorithm consists of the following steps:

Step 1: Symbol for the end of the plain text is added.

Step 2: Convert plain text to binary sequence using ASCII table.

Step 3: The pseudorandom generator based on Hitzl-Zele function is iterated forty eight times to produce 24 bits for $i$-value and 24 bits for $j$-value. These bits are converted modulo $n$ and modulo $m$, respectively. Integer values $i$ and $j$ are produced. 


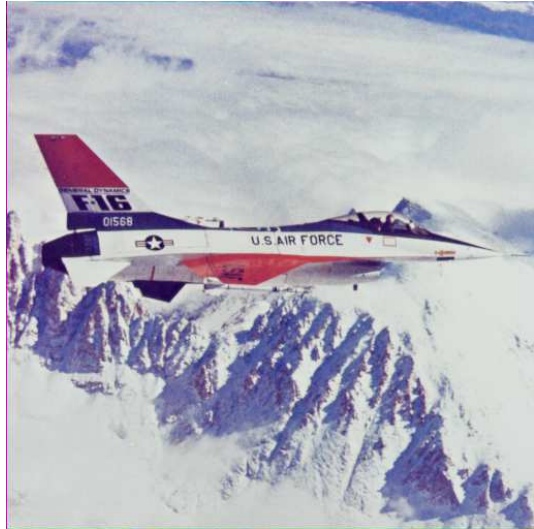

(a)

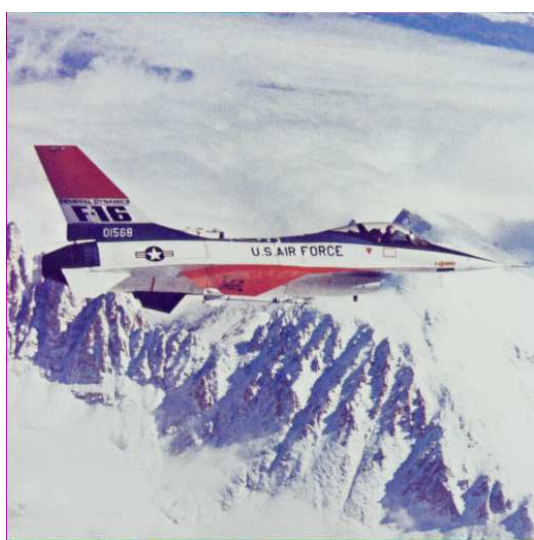

(d)

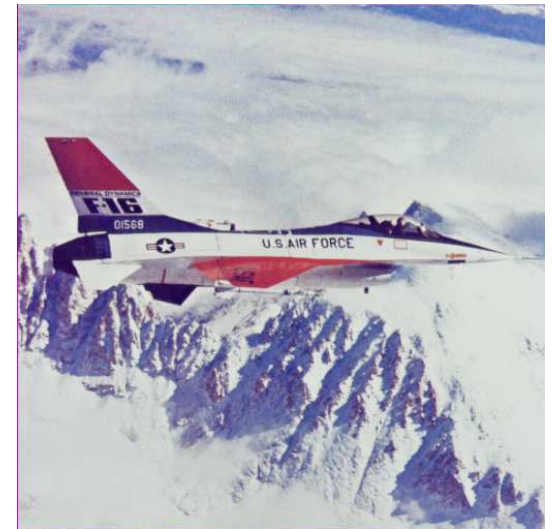

(b)

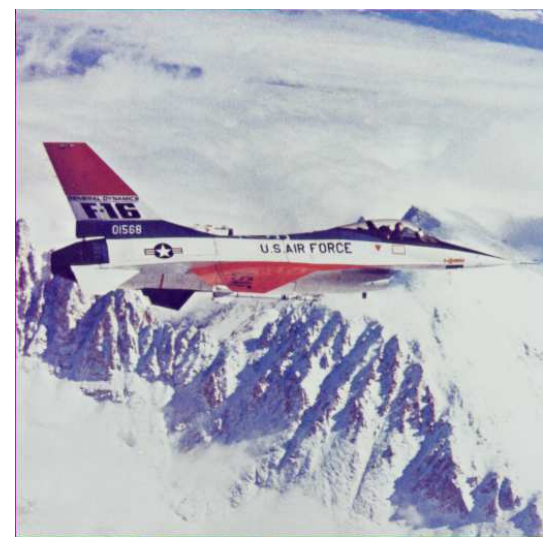

(e)

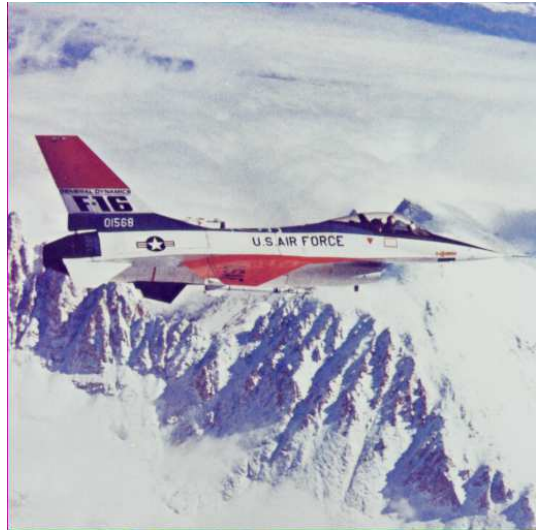

(c)

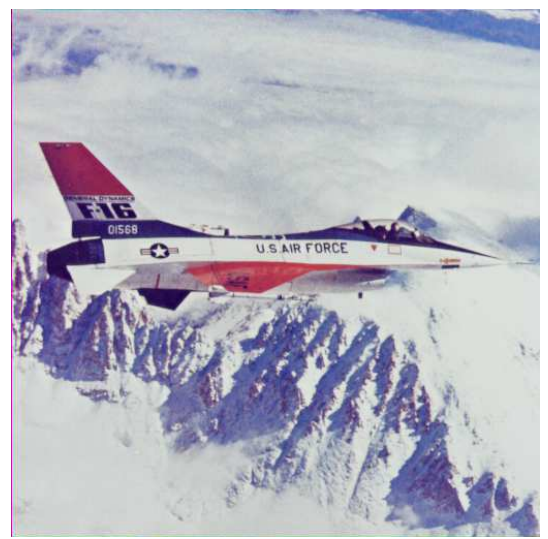

(f)

Fig. 3. Visual comparison of the 4.2.05 Airplane (F-16) plain image and the stego images: (a) original image, (b) stego image with 100 chars, (c) stego image with 200 chars, (d) stego image with 300 chars, (e) stego image with 400 chars, and (f) stego image with 500 chars.

TABLE IV

MSE AND PSNR FOR IMAGES WITH 100 CHARS (800 BITS), 200 CHARS (1600 BITS), 300 CHARS (2400 BITS), 400 CHARS (3200 BITS), AND 500 CHARS (4000 BITS) EMBEDDED.

\begin{tabular}{|c|c|c|c|c|c|c|c|c|c|c|}
\hline \multirow{2}{*}{ Images } & \multicolumn{2}{|c|}{ 100 chars } & \multicolumn{2}{c|}{ 200 chars } & \multicolumn{2}{c|}{ 300 chars } & \multicolumn{2}{c|}{ 400 chars } & \multicolumn{2}{c|}{ 500 chars } \\
\cline { 2 - 11 } & MSE & PSNR & MSE & PSNR & MSE & PSNR & MSE & PSNR & MSE & PSNR \\
\hline 4.1 .01 & 0.0021 & 74.9390 & 0.0041 & 71.9873 & 0.0061 & 70.2714 & 0.0079 & 69.1384 & 0.0101 & 68.0761 \\
\hline 4.1 .02 & 0.0021 & 74.9923 & 0.0041 & 71.9927 & 0.0061 & 70.2569 & 0.0082 & 68.9986 & 0.0101 & 68.0717 \\
\hline 4.1 .03 & 0.0021 & 74.9390 & 0.0041 & 72.0089 & 0.0061 & 70.2569 & 0.0082 & 69.0175 & 0.0102 & 68.0241 \\
\hline 4.1 .04 & 0.0021 & 74.9816 & 0.0041 & 72.0413 & 0.0062 & 70.2175 & 0.0081 & 69.0229 & 0.0102 & 68.0500 \\
\hline 4.1 .05 & 0.0020 & 75.0680 & 0.0040 & 72.1292 & 0.0061 & 70.2569 & 0.0082 & 68.9770 & 0.0103 & 67.9876 \\
\hline 4.1 .06 & 0.0020 & 75.0680 & 0.0041 & 71.9981 & 0.0061 & 70.2569 & 0.0082 & 68.9959 & 0.0103 & 68.0047 \\
\hline 4.1 .07 & 0.0020 & 75.2009 & 0.0040 & 72.0905 & 0.0059 & 70.3887 & 0.0081 & 69.0610 & 0.0101 & 68.0739 \\
\hline 4.1 .08 & 0.0020 & 75.1008 & 0.0041 & 72.0089 & 0.0061 & 70.2642 & 0.0082 & 69.0094 & 0.0102 & 68.0565 \\
\hline 4.2 .01 & 0.0005 & 80.7933 & 0.0011 & 77.8654 & 0.0016 & 76.2132 & 0.0021 & 74.9629 & 0.0026 & 73.9805 \\
\hline 4.2 .02 & 0.0005 & 80.9490 & 0.0011 & 77.7983 & 0.0016 & 76.1010 & 0.0021 & 74.9126 & 0.0026 & 73.9403 \\
\hline 4.2 .03 & 0.0006 & 80.7225 & 0.0011 & 77.8086 & 0.0016 & 76.1010 & 0.0021 & 74.9047 & 0.0026 & 73.9235 \\
\hline 4.2 .04 & 0.0005 & 81.0129 & 0.0010 & 78.1002 & 0.0015 & 76.3247 & 0.0020 & 75.0435 & 0.0025 & 74.0858 \\
\hline 4.2 .05 & 0.0005 & 81.0668 & 0.0010 & 78.0026 & 0.0015 & 76.3466 & 0.0020 & 75.0844 & 0.0025 & 74.1405 \\
\hline 4.2 .06 & 0.0006 & 80.6826 & 0.0010 & 77.9334 & 0.0016 & 76.2061 & 0.0021 & 74.9073 & 0.0026 & 73.9996 \\
\hline 4.2 .07 & 0.0005 & 80.9702 & 0.0010 & 78.0619 & 0.0015 & 76.2956 & 0.0020 & 75.0219 & 0.0026 & 74.0125 \\
\hline house & 0.0005 & 81.2215 & 0.0010 & 78.1609 & 0.0015 & 76.3797 & 0.0020 & 75.0734 & 0.0025 & 74.0728 \\
\hline
\end{tabular}

Step 4: Repeat Step 3 until unused pixel position $(i, j)$ is detected.

Step 5: Embed three bits from the input sequence into the last bit of the RED, GREEN and BLUE values of the selected pixel position $(i, j)$.

Step 6: Repeat Steps 3-5 until stego image is produced.
Decoding algorithm consists of the following steps:

Step 1: The pseudorandom generator based on Hitzl-Zele function is iterated forty eight times to produce 24 bits for $i$-value and 24 bits for $j$-value. These bits are converted modulo $n$ and modulo $m$, respectively. Integer values $i$ and $j$ are produced. 
TABLE V

PSNR (DB) OF THE PROPOSED ALGORITHM AND SOME OTHER ALGORITHMS

\begin{tabular}{|l|c|c|c|c|c|}
\hline Images & Ref. [2] & Ref. [4] & Ref. [17] & Ref. [18] & Proposed \\
\hline 4.2 .03 & 51.11 & 44.26 & 44.54 & 44.16 & 73.92 \\
\hline 4.2 .04 & 51.12 & 41.62 & 44.53 & 44.19 & 74.09 \\
\hline 4.2 .05 & 51.14 & 44.24 & 44.42 & - & 74.14 \\
\hline
\end{tabular}

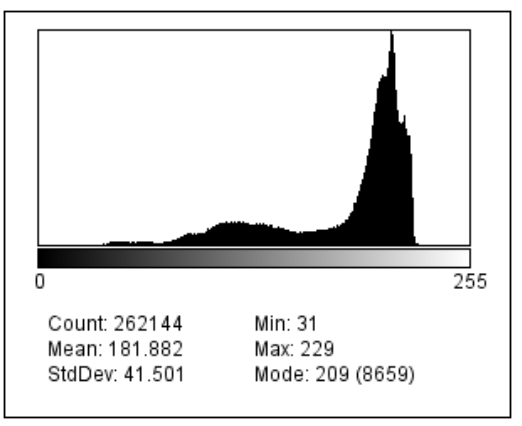

(a)

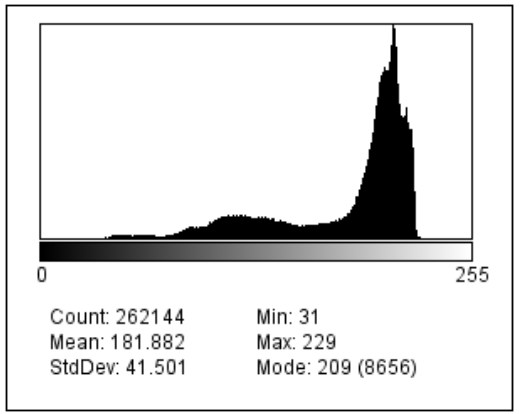

(d)

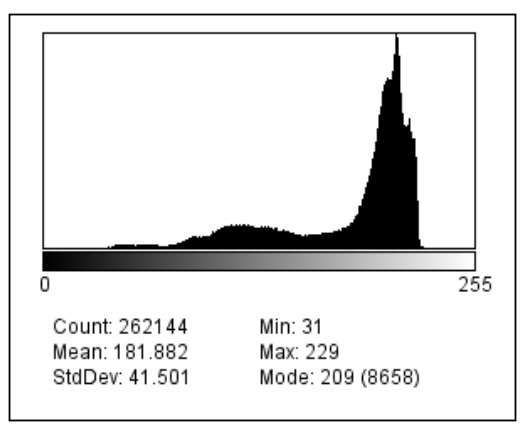

(b)

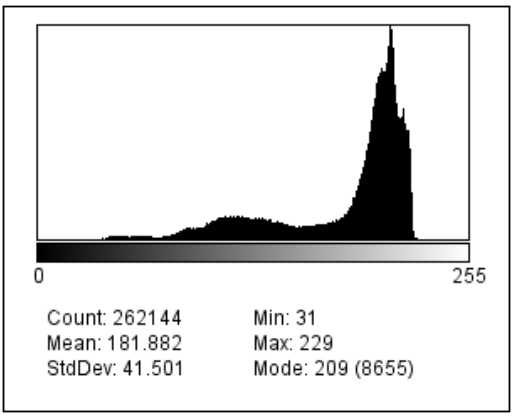

(e)

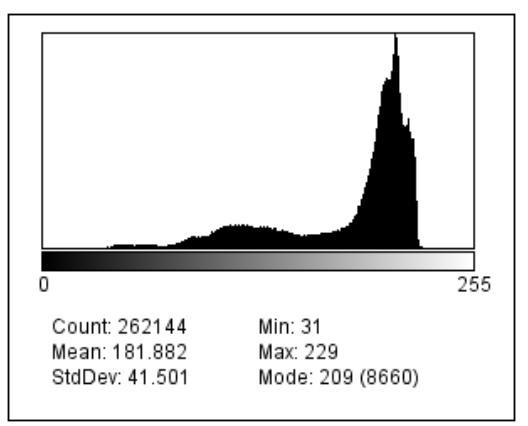

(c)

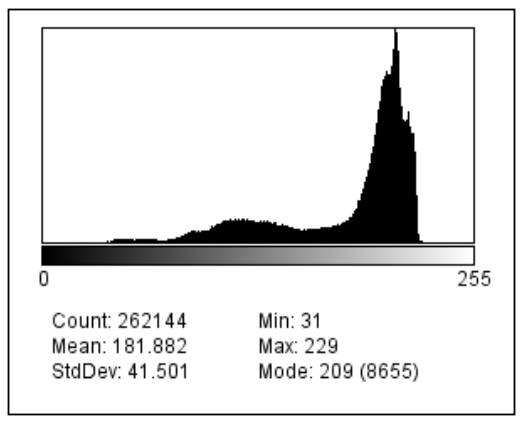

(f)

Fig. 4. Hystogram analysis of the 4.2.05 Airplane (F-16) plain image and the stego images: (a) original image, (b) stego image with 100 chars, (c) stego image with 200 chars, (d) stego image with 300 chars, (e) stego image with 400 chars, and (f) stego image with 500 chars.

Step 2: Repeat Step 1 until unused pixel position $(i, j)$ is detected.

Step 3: Retrieve three bits to the output binary sequence of the last bit of the RED, GREEN and BLUE values of the selected pixel position $(i, j)$.

Step 4: Every 8 bits are converted into char symbol and added to extracted text.

Step 5: Repeat Steps 1-4 until the end symbol is detected.

The designed LSB algorithm is implemented in $\mathrm{C}++$ programming language and the color images are selected from USC-SIPI image database (sipi.usc.edu/database/).

\section{B. Visual Inspection Analysis}

As an example, Figure 3 shows the image 4.2.05 Airplane (F16), Figure 3(a), and its stego images, Figure 3(b)-3(f). The visual inspection does not find differences between the plain image and the stego images. The images are identical optically with no indication of hidden messages.

\section{Peak Signal-to-Noise Ratio Analysis}

Peak Signal-to-Noise Ratio (PSNR) is the ratio between the maximum possible power of a signal and the power of noise contained in the signal. It is defined as follows:

$$
P S N R=10 \log _{10} \frac{\left(2^{d}-1\right)^{2}}{M S E}(d B),
$$

where $d$ is the bit depth of the pixel and MSE is the mean square error between the plain and stego images. MSE is defined as:

$$
M S E=\frac{1}{n n} \sum_{i=1}^{n} \sum_{j=1}^{n}\left(p[i, j]-p^{\prime}[i, j]\right)^{2},
$$

where $p[i, j], p^{\prime}[i, j]$ is the $i$ th-row $j$ th-column pixel in the plain and stego images, respectively.

In Table IV, we provide the computed values for MSE and PSNR for the proposed stego algorithm, where MSE and PSNR are calculated for images with 100 chars (800 bits), 200 chars (1600 bits), 300 chars (2400 bits), 400 chars (3200 bits), and 500 chars (4000 bits) embedded, are presented.

From the obtained results, Table IV, it is clear that the PSNR values are very high, above $68 \mathrm{~dB}$, which is an indication that the new LSB chaos-based steganography algorithm has a good level of security.

In Table $\mathrm{V}$, we have compare the PSNR of our embedding scheme with similar references [2], [4], [17], and [18]. 
Compared to other LSB steganography schemes, we can see that proposed scheme has higher PSNR values.

\section{Histogram Analysis}

Image histograms are graphical representation of the tonal distribution in digital images. This test compares histograms of plain and stego images. In addition, histogram analysis was performed using ImageJ (https://imagej.nih.gov/ij/) histogram analysis of the distribution of gray values in the cover and the stego image 4.2.05 Airplane (F16), see Figure 4. It is examined that the histograms of the stego images are very similar to that of the plain image with no indication of hidden messages in stego images.

\section{FUTURE WORK}

In the future steps, we intend to experiment with hiding messages not only in least significant bit in order to increase container capacity without any sign of steganography. We also intend to use Bent boolean functions to increase chaos behaviour and pseudorandomness of PRG. Hardware implementation of the proposed algorithm is one of our goals.

\section{CONCLUSION}

Novel least significant bit steganography algorithm based on a Hitzl-Zele chaotic function, is designed. Empirical experiments are provided for testing the security of the proposed scheme. Our stego analysis, visual inspection, peak signalto-noise ratio, and histogram analysis, show that the novel algorithm has enough key size, high key sensitivity and excellent performance.

\section{ACKNOWLEDGMENT}

The authors are grateful to the anonymous referees for valuable and helpful comments, editorial board and support of INTL Journal of Electronics and Telecommunications.

\section{REFERENCES}

[1] Alvarez, G., Li, S. (2006) Some Basic Cryptographic Requirements for Chaos-Based Cryptosystems, International Journal of Bifurcation and Chaos, 16, 2129 - 2151

[2] Amirtharajan, R., Rayappan, J.B.B. (15 June 2012), An intelligent chaotic embedding approach to enhance stego-image quality, Information Sciences, Volume 193, Pages 115-124

[3] M. Andrecut, Logistic Map as a Random Number Generator, International Journal of Modern Physics B, 12 (1999), 921 - 930.

[4] Aziz, M., Tayarani-N, M.H., and Afsar, M. (2015), A cycling chaosbased cryptic-free algorithm for image steganography, Nonlinear Dynamics, 80 (3), 1271-1290

[5] Dăscălescu, A., Boriga, R.E., Diaconu, A., Study of a New Chaotic Dynamical System and Its Usage in a Novel Pseudorandom Bit Generator, Mathematical Problems in Engineering, 2013 (2013), Article ID 769108, 1-10.

[6] Diaconu, A.V. (2015), A Parallel Architecture Design for Ultra-Fast Image Encryption within WMSNS, Proceedings of the Romanian Academy, Series A 16 (SI), 313-320.

[7] François, M., D. Defour, P. Berthomé, A Pseudo-Random Bit Generator Based on Three Chaotic Logistic Maps and IEEE 754-2008 FloatingPoint Arithmetic, Lecture Notes in Computer Science, 8402 (2014), 229 -247 .
[8] François, M., T. Grosges, D. Barchiesi, R. Erra, Pseudo-Random Number Generator Based on Mixing of Three Chaotic Maps, Communications in Nonlinear Science and Numerical Simulation, 19 (2014), 887 . 895.

[9] Fridrich, J. (1998), Symmetric Ciphers Based on Two-Dimensional Chaotic Maps. Int. J. Bifurcation Chaos, 8, 1259-1284.

[10] Hitzl, D.L., Zele, F. (1985), An exploration of the Hénon quadratic map, Physica D: Nonlinear Phenomena, Volume 14, Issue 3, Pages 305-326

[11] Marsaglia, G. DIEHARD: a Battery of Tests of Randomness, http://www. fsu.edu/pub/diehard/.

[12] Nassar, S.S., Ayad, N.M., Kelash, H.M., El-sayed, H.S., El-Bendary, M.A.M. Abd El-Samie, F.E., Faragallah, O.S. (2016), Secure Wireless Image Communication Using LSB Steganography and Chaotic Baker, Ciphering Wireless Personal Communications, 91 (3), pp. 1023-1049.

[13] Nithin Kumar, S.S.V., Charan, G.S., Karthikeyan, B., Vaithiyanathan, V. Rajasekhar Reddy, M. (2016), A hybrid approach for data hiding through chaos theory and reversible integer mapping, Advances in Intelligent Systems and Computing, 412, pp. 483-492.

[14] Parvees, M.Y.M., Samath, J.A., Kaspar Raj, I., Madhavan Nirmal, R. (2017), Chaos-based steganocryptic approach to protect medical images with text data of patients, Journal of Medical Imaging and Health Informatics, 7 (1), pp. 118-125.

[15] Pichler, F. and Scharinger, J. (1995), Ciphering by Bernoulli-shifts in finite Abelian groups, Contributions to General Algebra, Vol. 9, eds. Kaiser, H.K., Muller, W.B. and Pilz G.F., A-4040 Linz, 249-256.

[16] Pichler, F. and Scharinger, J. (1995), Finite dimensional generalized Baker dynamical systems for cryptographic applications, Lecture Notes in Computer Science, Vol. 1030, 465-476.

[17] Rajendran, S., Doraipandian, M. (2017), Chaotic Map Based Random Image Steganography Using LSB Technique, International Journal of Network Security, Vol.19, No.4, 593-598.

[18] Ranjith Kumar, R., Jayasudha, S., Pradeep, S. (2016), Efficient and secure data hiding in encrypted images, A new approach using chaos Information Security Journal, 25 (4-6), pp. 235-246.

[19] Saha, P., Stragatz, S.H. (1995), The Birth of Period Three, Mathematics Magazine, 68 (1), pp. 42-47.

[20] Saturday, J.C., Udofia, K.M. and Jimoh, A.J. (2016), Design of Dual Band Microstrip Antenna Using Reactive Loading Technique, Mathematical and Software Engineering, 2 (2), 114-121.

[21] Rukhin, A., J. Soto, J. Nechvatal, M. Smid, E. Barker, S. Leigh, M. Levenson, M. Vangel, D. Banks, A. Heckert, J. Dray, and S. Vo (2010), "A Statistical test suite for random and pseudorandom number generators for cryptographic application," NIST Special Publication 800 22, Revision 1a (Revised: April 2010), Lawrence E. Bassham III http://csrc.nist.gov/rng/.

[22] Stoyanov, B., Kordov, K., Novel Image Encryption Scheme Based on Chebyshev Polynomial and Duffing Map, The Scientific World Journal 2014, Article ID 283639, 2014, 1-11.

[23] Stoyanov, B., Kordov, K., A Novel Pseudorandom Bit Generator Based on Chirikov Standard Map Filtered with Shrinking Rule, Mathematical Problems in Engineering 2014, Article ID 986174, 2014, 1-4.

[24] Stoyanov, B., Kordov, K., A Novel Secure Pseudo-Random Number Generation Scheme Based on Two Tinkerbell Maps, submitted, under review.

[25] Stoyanov, B., Kordov, K., Novel Zaslavsky Map Based Pseudorandom Bit Generation Scheme, Applied Mathematical Sciences, Vol. 8, 2014 no. 178, 8883-8887, http://dx.doi.org/10.12988/ams.2014.410879.

[26] Stoyanov, B.P., Zhelezov, S.K., Kordov, K.M. (2016), Least significant bit image steganography algorithm based on chaotic rotation equations, Comptes Rendus de L'Academie Bulgare des Sciences, 69 (7), 845-850.

[27] Sun, F., Liu, S., Cryptographic pseudo-random sequence from the spatia chaotic map, Chaos, Solitons \& Fractals, 41 (2009), 2216 - 2219.

[28] Walker, J., ENT: a pseudorandom number sequence test program, http: //www.fourmilab.ch/random/.

[29] Yang, C, Liu, F., Lian, S., Luo, X., Wang, D. (2012), Weighted StegoImage Steganalysis of Messages Hidden into Each Bit Plane. Comput J., 55, 717-727.

[30] IEEE Computer Society (2008), 754-2008 - IEEE Standard for Floating-Point Arithmetic, Revision of ANSI/IEEE Std 754-1985, DOI: 10.1109/IEEESTD.2008.4610935. 